\title{
Continuous Improvement Practices in Manufacturing Companies in the Sultanate of Oman
}

\author{
Samiya Abdullah Al-Qayoudhi', Shanmuga Pria², Venkata Vara Prasad ${ }^{3}$ \\ University of Technology and Applied Sciences, Shinas \\ Email: ${ }^{1}$ Rawan6660@yahoo.com, ${ }^{2}$ Dr.s.pria@gmail.com, ${ }^{3}$ Venkata.prasad@ @hct.edu.om
}

Article History: Received on $11^{\text {th }}$ Apr. 2021, Revised on $6^{\text {th }}$ May. 2021, Published on $4^{\text {th }}$ Jun. 2021

\begin{abstract}
Purpose: The aims of this study were to identify Continuous Improvement (CI) practices prevailing in the manufacturing companies with regards to Visual Management, Employee Engagement, Leadership, and Risk Management; to identify continuous improvement tools that have been adopted by the manufacturing companies, and to analyze the critical success factors for implementing CI practices in manufacturing companies.

Design/methodology/approach: For this study, a survey questionnaire was used to collect the CI practices information from manufacturing companies in Oman. 146 samples were collected from 75 manufacturing units selected at random from the Public Establishment for Industrial Estate database. The data was analyzed for reliability, robustness, ranking tests using Statistical Package for Social Statistics (SPSS).

Findings: The majority of the manufacturing firms were using only the CI program for the past five years. However, there were not substantial cost savings for these businesses. The overall sales increased, while the average processing time had declined and the product recalls also had increased.

Research limitations/implications: The most important factors identified for the effective implementation of CI in industrial firms were effective communication, top management encouragement, and employee involvement, and a conducive organizational atmosphere.

Social implications: Sultanate of Oman like any other GCC countries is undergoing a critical phase in the economy because of the oil price plunge combined with the ongoing COVID-19 pandemic. The paper will be of use to academics, researchers, and continuous improvement practitioners.

Originality/Value: So far, very few studies have been conducted in analyzing the factors correlated to CI in the Omani context and no study was carried out before in exploring the CI application in the Sultanate of Oman.
\end{abstract}

Keywords: Continuous Improvement Practices, Critical Success Factors, Visual Management, Employee Engagement, Leadership, Risk Management, Manufacturing Companies/Industries in Oman.

\section{Introduction}

Continuous Improvement (CI) is a quality management concept that was introduced in Japan and has spread all over the world. CI is one of the pillars of production processes focusing on total quality management (TQM), lean method of production, and other classic manufacturing models. A collection of activities that make up a process aimed at improving efficiency is known as Continuous Improvement. These practices primarily involve the reduction/elimination of unwanted wastage, during production. Lower maintenance expenses, the long life of the equipment, and low average expansion costs are all the advantages of effective CI deployment programs in an enterprise. Caffyn (1999) described CI as an approach that focuses on gradual innovation and includes the whole enterprise. CI can be characterized as a standard process that maximizes output through detecting and removing waste (Dossenbach, 2006).

CI method, though often correlated with these models, can be applied as a stand-alone initiative that results in cumulative changes in organizational performance metrics. CI is made up of three things, namely paths, place, and phase (Bessant and Francis, 1999). The first two are concerned with the organization's ability to accumulate skills and their proper time usage. The third component is organizational routine, which decides how to innovate, learn, and renew.

Atkinson (1994) suggested the following components for change which the management needs to focus namely commitment of the management, awareness, application, benchmarking, appreciation, and regeneration. Dabhilkar and Bengtsson (2004) argued that given the committed team of managers, the use of TQM techniques for efficient problem solving becomes easier and strategy implementation brings out 
operations efficiency through continuous improvements. Al-Khawaldeh and Sloan (2007) revealed that implementation of continuous improvement activities in companies come across problems related to measuring the performances, financial support, and organizational commitment.

The global market climate is marked by severe competition and increased uncertainty in consumer needs. These developments have left their indelible imprints on various aspects of the manufacturing industry. Due to intensified global competitiveness, all businesses are focused on increasing profits through system simplification and gradual upgrades leveraging new technology. Continuous improvement of production practices is important for the businesses to face the competition, maintain their stake in the global market and local market as well (Singh and Singh, 2018).

The Sultanate of Oman like any other GCC countries is undergoing a critical phase in the economy because of the oil price plunge combined with the ongoing COVID-19 pandemic. The inflation report released on March 2021 reviews the most inflation rates during the month, and price movement in the various provinces. The inflation rate in Oman has been recorded at 3.4 percent an increase from 1 percent in 2020 . Since oil prices have gone down and also inflation runs in the economy it is necessary for the manufacturing companies to adopt ways to reduce cost and improve productivity.

Production drives estimated as the key performance indicators ranked 45 out of 100 in 2018 (World Economic Forum, 2018). This will be supporting Oman to become one of the top 20 countries by 2030 (Oman Observer, 2020). Sustainable development of the manufacturing industry in Oman relies mainly on the increase in net exports. Therefore, the Ministry of Commerce and Industry (MOCI) in Oman has introduced trade policies accordingly. With the policies targeting the manufacturing sector, import replacements and export recommendation is necessitated. Manufacturing industries that are competitive in the domestic market will export more. So, CI practices at this juncture gain a lot of attention. CI helps in refining the production practice, reducing the cost of production, etc., and make the price reasonable in the local markets and outside the country.

CI can be achieved through Kaizen techniques which were proven by Japanese experiments. Kaizen is so popular in the western world. The term refers to a method of incrementally improving the normal method of working overtime (Chen et al., 2001). Kai denotes transition and Zen denotes goodness. The word is derived from the Japanese term Gemba Kaizen, which means 'continuous development and perfection' (Yanjiang et al., 2006).

The application or incorporation of CI methods or strategies is dependent on several factors. Fewer experiments have been performed in the Omani context to investigate the factors associated with CI. So far, very few studies have been conducted in analyzing the factors correlated to CI in the Omani context and no study was carried out before in exploring the CI application in the Sultanate of Oman. This is the prime reason for undertaking the study and this study adds value to manufacturing industries in Oman.

\section{Research Questions}

The research questions of the study were:

1. What are the CI practices prevailing in the manufacturing companies with regards to Visual Management, Employee Engagement, Leadership, and Risk Management?

2. Which continuous improvement tools have been adopted by the manufacturing companies?

3. What are the critical success factors for implementing CI practices in manufacturing companies?

\section{Research Objectives}

The objectives of the study were:

1. To identify CI practices prevailing in the manufacturing companies with regards to Visual Management, Employee Engagement, Leadership, and Risk Management.

2. To identify continuous improvement tools that have been adopted by the manufacturing companies.

3. To analyze the critical success factors for implementing CI practices in the manufacturing companies 


\section{Review of Literature}

Continuous Improvement is an effective strategic instrument for increasing organizational productivity (Marin-Garcia et al., 2008). Lean is one of the popular best continuous improvement practices (Welo \& Ringen, 2015). CI can be linked to a variety of problems, including organization of improvement teams (Augsdorfer and Harding, 1995; Dabhilkar and Bengtsson, 2004), the capacity of the improvement team (Bessant and Francis, 1999; Jabnoun, 2001; Lee, 2004; Abrahamsson and Gerdin, 2006), the association between CI and TQM (Chapman and Hyland, 1997; Hyland et al., 2000), feedback structures (Delbridge and Barton, 2002), as well as performance metrics and CI operations' supporting systems (Bechet, 2000).

Although an efficient quality improvement program is one of the primary conditions for achieving and maintaining operational performance in a competitive environment, shortcomings in executing effective continuous improvement plans surpass achievements (Anand et al., 2009; De Morais et al., 2012).

\section{Visual Management (VM)}

Many organizations face major challenges in providing the right information to the concerned people at the appropriate moment efficiently so that the best decisions are made for suitable actions (Harris and Harris, 2008; Tezel et al., 2010). VM acknowledges that the concept lets shop floor staff and supervisors understand manufacturing processes by simply visualizing the importance of their jobs in a responsive manner (Tezel et al., 2016).

\section{Employee Engagement}

Employee engagement is a kind of strategy for the sustainability of the organization in the long run through mutual commitment between the employees and the organization (Taneja et al., 2015). Employee engagement entails communicating the value of CI to workers as well as defining mutual objectives that are shared by all employees. This includes encouraging training, leadership, collaboration, or the establishment of contact networks to access and disseminate information and expertise (Escrig-Tena, 2004). The pioneer Lean manufacturing system involved continuous employee participation and engagement (Maskell et al., 2004) whereas in Kaizen, management keeps improving the standard of the processes for better employee empowerment, etc., without making more investment (Liker \& Hoseus, 2008).

\section{Leadership}

In the introduction of continuous process improvement, senior leaders play a vital role (Das et al., 2011;

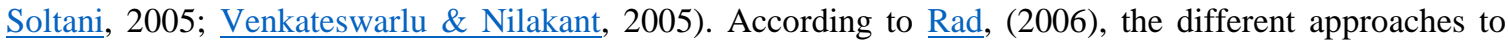
continuous improvement necessitate the support of the senior management and involvement and the employees of such organization will least consider such continuous improvement if it is incompatible with the organization's culture.

\section{Risk Management (RM)}

Risk management of a project is a controlled process that involves the identification of various types of risks which emerge and responding to such risks (Galli \& Kaviani, 2017). In an organization, risk management involves deciding which risks to be minimized and which risks should be considered (Danciulescu, 2013). Risk management aims to reduce perils and increase the possibility of good events in an organization (Choi et al., 2016). Companies should reduce the risk levels that might emerge and risk management will be the critical success factor to overcome the same (Galli, 2018). Companies use risk management to enhance their products in comparison to rivals as RM emphasizes the removal of waste and the reduction in costs (Avanesov, 2009).

From the above review of literature, most of the measurements were drawn. Thus, these instruments utilized in this study were adopted contextualizing to Omani manufacturing industries. The respondents rated the measurement items - 20 critical success factors (CSFs) on a scale of 1 to 5 (Likert Scale). Those CSFs were derived from the above literature of $\mathrm{CI}$ as shown in Table 1. 
Table 1 Measurement of Constructs

\begin{tabular}{|c|c|c|c|}
\hline \# & Instruments & Statements & Authors \\
\hline 1 & Visual Management & 5 & Tezel et al. (2016) \\
\hline 2 & $\begin{array}{l}\text { Employee } \\
\text { Engagement }\end{array}$ & 5 & Escrig-Tena (2004) \\
\hline 3 & Leadership & 5 & $\begin{array}{l}\text { Das et al. (2011); Soltani }(2005) ; \\
\text { Talib et al. }(2011) ; \underline{\text { Venkateswarlu }} \\
\underline{\text { \& Nilakant }}(2005) ; \underline{\operatorname{Rad}}(2006)\end{array}$ \\
\hline 4 & Risk management & 5 & Danciulescu (2013) \\
\hline
\end{tabular}

\section{Research Methodology}

Based on the above literature review, a questionnaire was prepared which is meant to provide the basis for CI practices of the manufacturing companies in Oman. It included the following parts viz.

1. Demographic information

2. Manufacturing companies experience with CI tools and techniques

3. Company success factors

4. Critical success factors for CI implementation in manufacturing companies in Oman.

The survey was sent to 75 manufacturing units selected at random from the Public Establishment for Industrial Estate database and 146 completed questionnaires were obtained out of 200 questionnaires mailed. The data was analyzed for reliability, robustness, ranking tests using Statistical Package for Social Statistics (SPSS).

\section{Findings}

Cronbach alpha score was 0.81 passes the minimum requirement (Nunnally, 1960).

Table 2 Demographic details of the respondents

\begin{tabular}{|c|c|c|c|}
\hline Characteristics & & Frequency & $\%$ \\
\hline \multirow{2}{*}{ Gender } & Male & 83 & 56.8 \\
\hline & Female & 63 & 43.2 \\
\hline \multirow{5}{*}{ Age } & $18-25$ & 13 & 8.9 \\
\hline & $26-35$ & 88 & 60.3 \\
\hline & $36-45$ & 40 & 27.4 \\
\hline & $46-55$ & 4 & 2.7 \\
\hline & $56-60$ & 1 & 0.7 \\
\hline \multirow{5}{*}{ Designation } & President & 6 & 4.1 \\
\hline & Vice President & 15 & 10.3 \\
\hline & Director/Manager & 16 & 11.0 \\
\hline & Supervisor/Foreman & 39 & 26.7 \\
\hline & Operator/Technician & 70 & 47.9 \\
\hline \multirow{5}{*}{$\begin{array}{l}\text { Experience in } \\
\text { the Current } \\
\text { Position }\end{array}$} & $0-5$ years & 75 & 51.4 \\
\hline & $6-10$ years & 45 & 30.8 \\
\hline & $11-15$ years & 15 & 10.3 \\
\hline & $16-20$ years & 9 & 6.2 \\
\hline & 21-25 years & 2 & 1.4 \\
\hline \multirow{6}{*}{$\begin{array}{l}\text { Current } \\
\text { Department }\end{array}$} & Corporate & 23 & 15.8 \\
\hline & Quality & 12 & 8.2 \\
\hline & Manufacturing & 26 & 17.8 \\
\hline & Logistics & 7 & 4.8 \\
\hline & Finance & 14 & 9.6 \\
\hline & Marketing & 6 & 4.1 \\
\hline
\end{tabular}




\begin{tabular}{|c|c|c|c|}
\hline & Sales & 4 & 2.7 \\
\hline & Regulatory & 1 & 0.7 \\
\hline & Others & 53 & 36.3 \\
\hline \multirow{5}{*}{$\begin{array}{l}\text { Number of } \\
\text { Employees }\end{array}$} & $0-100$ & 39 & 26.7 \\
\hline & $101-300$ & 25 & 17.1 \\
\hline & $301-500$ & 18 & 12.3 \\
\hline & $501-1000$ & 29 & 19.9 \\
\hline & More than 1000 & 35 & 24.0 \\
\hline \multirow{7}{*}{$\begin{array}{lr}\text { CI } & \text { Critical } \\
\text { Success } & \text { Factors } \\
\text { of } & \text { the } \\
\text { companies } & \end{array}$} & Visual Management only & 6 & 4.1 \\
\hline & Employee Engagement only & 6 & 4.1 \\
\hline & Risk Management only & 28 & 19.2 \\
\hline & Leadership only & 19 & 13.0 \\
\hline & $\begin{array}{l}\text { Employee Engagement \& } \\
\text { Leadership }\end{array}$ & 14 & 9.6 \\
\hline & $\begin{array}{l}\text { Visual Management, Risk } \\
\text { Management \& Leadership }\end{array}$ & 6 & 4.1 \\
\hline & All the four & 67 & 45.9 \\
\hline \multirow{7}{*}{$\begin{array}{l}\text { Duration of the } \\
\text { use of CI tools } \\
\text { and techniques }\end{array}$} & $0-5$ years & 60 & 41.1 \\
\hline & $6-10$ years & 45 & 30.8 \\
\hline & 11-15 years & 26 & 17.8 \\
\hline & $16-20$ years & 7 & 4.8 \\
\hline & 21-25 years & 1 & 0.7 \\
\hline & More than 25 years & 1 & 0.7 \\
\hline & Did not use any CI & 6 & 4.1 \\
\hline \multirow{3}{*}{$\begin{array}{l}\text { CI program site } \\
\text { / Corporate } \\
\text { initiative }\end{array}$} & Site Specific & 50 & 34.2 \\
\hline & Corporate Initiative & 82 & 56.2 \\
\hline & Not applicable & 14 & 9.6 \\
\hline \multirow{3}{*}{ Average Sales } & Increased & 98 & 67.1 \\
\hline & Constant & 37 & 25.4 \\
\hline & Decreased & 11 & 7.5 \\
\hline \multirow{3}{*}{$\begin{array}{l}\text { Average Cycle } \\
\text { time }\end{array}$} & Decreased & 85 & 58.2 \\
\hline & Increased & 27 & 18.5 \\
\hline & Not sure & 34 & 23.3 \\
\hline \multirow{3}{*}{$\begin{array}{l}\text { Products } \\
\text { Recalled }\end{array}$} & Yes & 67 & 45.9 \\
\hline & No & 48 & 32.9 \\
\hline & Not sure & 31 & 21.2 \\
\hline \multirow{19}{*}{$\begin{array}{l}\text { Type of } \\
\text { Products }\end{array}$} & Aluminium & 5 & 3.5 \\
\hline & Steel & 4 & 2.8 \\
\hline & Building Materials & 10 & 6.9 \\
\hline & Cement & 2 & 1.4 \\
\hline & Quick Lime & 1 & 0.7 \\
\hline & Ceramic & 2 & 1.4 \\
\hline & Chemicals & 1 & 0.7 \\
\hline & Electrical Switch Board & 4 & 2.8 \\
\hline & Oil \& Gas & 35 & 24.5 \\
\hline & Food & 3 & 2.1 \\
\hline & Iron-ore and pellets & 2 & 1.4 \\
\hline & Paper & 1 & 0.7 \\
\hline & Plastics & 1 & 0.7 \\
\hline & Methanol Gas & 3 & 2.1 \\
\hline & Propylene & 1 & 0.7 \\
\hline & Polymers & 1 & 0.7 \\
\hline & Bottles & 2 & 1.4 \\
\hline & Sulphuric Acid & 3 & 2.1 \\
\hline & Others & 65 & 43.4 \\
\hline
\end{tabular}

Source: Questionnaire 
Table 3 CI Tools \& Techniques used by the companies

\begin{tabular}{|l|c|c|}
\hline \multicolumn{1}{|c|}{ CI Tools } & Frequency & \% \\
\hline Kaizen & 40 & 27.4 \\
\hline Six Sigma & 34 & 23.3 \\
\hline Design Excellence & 23 & 15.7 \\
\hline Lean & 26 & 17.8 \\
\hline TQM & 16 & 11.0 \\
\hline HACCP & 7 & 4.8 \\
\hline
\end{tabular}

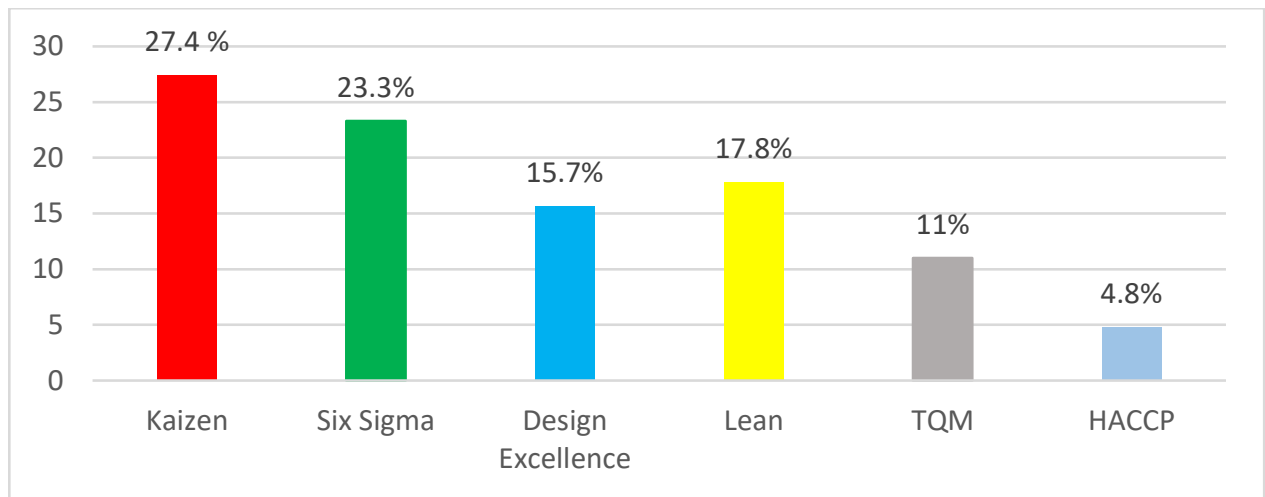

Figure 1 CI Tools \& Techniques used by the companies

Figure 1 shows the various CI tools and techniques used to ensure successful implementation of CI in their companies and the popular tool among the respondents i.e. Kaizen.

Table 4 Visual Management (VM) Tools used by the companies

\begin{tabular}{|l|c|c|}
\hline \multicolumn{1}{|c|}{ Visual Management Tools } & Frequency & \% \\
\hline Cross Training & 49 & 33.5 \\
\hline 5 S & 23 & 15.7 \\
\hline Control Plans & 12 & 8.2 \\
\hline Failure Mode and Effect Analysis & 15 & 10.3 \\
\hline Standard Operating Procedures & 28 & 19.2 \\
\hline Kanban & 9 & 6.2 \\
\hline Dashboards & 10 & 6.9 \\
\hline
\end{tabular}

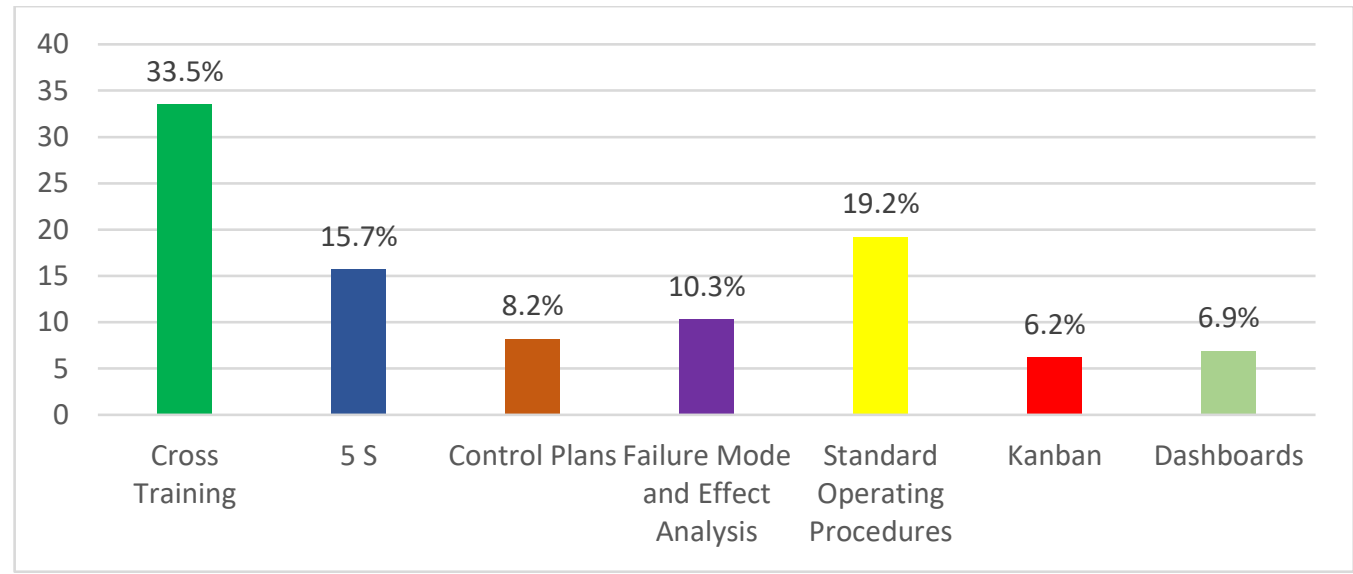

Figure 2 Visual Management (VM) Tools used by the companies 
Figure 2 shows the various VM tools used to ensure successful implementation of CI in their companies and the popular tool among the respondents i.e. Cross Training.

Table 5 Visual Management (VM) Tools

\begin{tabular}{|c|c|c|c|c|c|c|c|c|}
\hline Statements & SD & D & $\mathbf{N}$ & A & SA & $\begin{array}{c}\text { KS } \\
\text { value }\end{array}$ & $\chi^{2}$ & $\begin{array}{c}\mathbf{p} \\
\text { value }\end{array}$ \\
\hline $\begin{array}{l}\text { 1. Top management } \\
\text { supports } \\
\text { implementation the } \\
\text { VM }\end{array}$ & $\begin{array}{c}36 \\
24.7 \%\end{array}$ & $\begin{array}{c}35 \\
24.0 \%\end{array}$ & $\begin{array}{c}42 \\
28.7 \%\end{array}$ & $\begin{array}{c}20 \\
13.7 \%\end{array}$ & $\begin{array}{c}13 \\
8.9 \%\end{array}$ & .166 & \multirow{5}{*}{68.931} & \multirow{5}{*}{.000} \\
\hline $\begin{array}{l}\text { 2. VM tools allows } \\
\text { you to detect the } \\
\text { deviations from the } \\
\text { standards }\end{array}$ & $\begin{array}{c}32 \\
21.9 \%\end{array}$ & $\begin{array}{c}40 \\
27.4 \%\end{array}$ & $\begin{array}{c}44 \\
30.1 \%\end{array}$ & $\begin{array}{c}23 \\
15.8 \%\end{array}$ & $\begin{array}{c}7 \\
4.8 \%\end{array}$ & .176 & & \\
\hline $\begin{array}{l}3 . \quad \text { VM strategy } \\
\text { enables the flow of } \\
\text { information }\end{array}$ & $\begin{array}{c}31 \\
21.2 \%\end{array}$ & $\begin{array}{c}32 \\
21.9 \%\end{array}$ & $\begin{array}{c}53 \\
36.3 \%\end{array}$ & $\begin{array}{c}21 \\
14.4 \%\end{array}$ & $\begin{array}{c}9 \\
6.2 \%\end{array}$ & .201 & & \\
\hline $\begin{array}{l}\text { 4. VM facilitates } \\
\text { distribution } \quad \text { of } \\
\text { responsibilities among } \\
\text { employees }\end{array}$ & $\begin{array}{c}36 \\
24.7 \%\end{array}$ & $\begin{array}{c}36 \\
24.7 \%\end{array}$ & $\begin{array}{c}45 \\
30.8 \%\end{array}$ & $\begin{array}{c}22 \\
15.0 \%\end{array}$ & $\begin{array}{c}7 \\
4.8 \%\end{array}$ & .176 & & \\
\hline $\begin{array}{l}\text { 5. VM leads to } \\
\text { providing the best } \\
\text { solution }\end{array}$ & $\begin{array}{c}32 \\
21.9 \%\end{array}$ & $\begin{array}{c}30 \\
20.5 \%\end{array}$ & $\begin{array}{c}49 \\
33.6 \%\end{array}$ & $\begin{array}{c}26 \\
17.8 \%\end{array}$ & $\begin{array}{c}9 \\
6.2 \%\end{array}$ & .193 & & \\
\hline
\end{tabular}

Null Hypothesis.1: No relationship exists between the VM tools and the choices of the respondents.

Table 5 shows p-value $<0.05$. So null hypothesis 1 got rejected. i.e., it is understood that there exists a significant relationship between the VM tools and the choices of the respondents. From K-S values of Kolmogorov-Smirnov test, 'VM strategy enables the flow of information' ranked first (0.201); 'VM leads to providing the best solution' ranked second (0.193) and both 'VM tools allow you to detect the deviations from the standards' and 'VM facilitates the distribution of responsibilities among employees' ranked third (0.176).

Table 4 Ways of Employee Engagement used by the companies

\begin{tabular}{|l|c|c|}
\hline \multicolumn{1}{|c|}{ Ways of Employee Engagement } & Frequency & \% \\
\hline Appreciation \& Recognition & 54 & 37.0 \\
\hline Training \& Development Programs & 22 & 15.1 \\
\hline Work Schedule Flexibility & 12 & 8.2 \\
\hline Creating Opportunities outside the workplace & 12 & 8.2 \\
\hline $\begin{array}{l}\text { Attending Industry Tradeshows/Virtual Events } \\
\text { with Employees }\end{array}$ & 12 & 8.2 \\
\hline Encouraging employees to be creative/innovative & 12 & 8.2 \\
\hline Hiring the right people for the right job & 22 & 15.1 \\
\hline
\end{tabular}




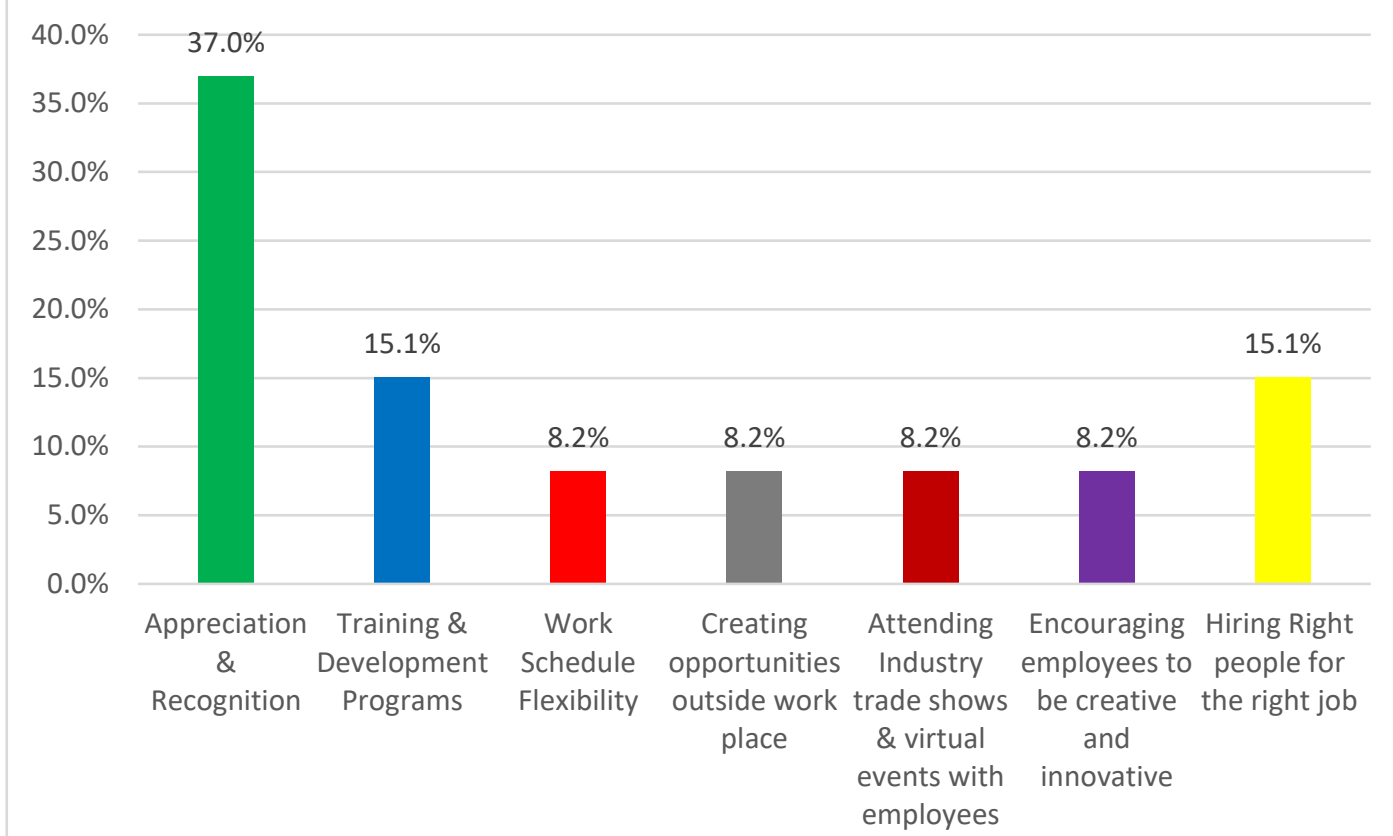

Figure 3 Ways of Employee Engagement used by the companies

Figure 3 shows the various ways of Employee engagement used to ensure successful implementation of CI in their companies and the popular means among the respondent companies was Appreciation \& Recognition.

Table 6 Employee Engagement

\begin{tabular}{|c|c|c|c|c|c|c|c|}
\hline Statements & SD & D & $\mathbf{N}$ & $\mathbf{A}$ & SA & $\chi^{2}$ & $\begin{array}{c}\text { p- } \\
\text { value }\end{array}$ \\
\hline $\begin{array}{l}\text { 1. The top management } \\
\text { supports employees to } \\
\text { achieve continuous } \\
\text { improvement }\end{array}$ & $\begin{array}{c}29 \\
19.9 \%\end{array}$ & $\begin{array}{c}29 \\
19.9 \%\end{array}$ & $\begin{array}{c}47 \\
32.1 \%\end{array}$ & $\begin{array}{c}23 \\
15.8 \%\end{array}$ & $\begin{array}{c}18 \\
12.3 \%\end{array}$ & 17.379 & .002 \\
\hline $\begin{array}{l}2 . \text { The } \\
\text { provanides } \\
\text { training }\end{array}$ & $\begin{array}{c}31 \\
21.2 \%\end{array}$ & $\begin{array}{c}35 \\
24.0 \%\end{array}$ & $\begin{array}{c}41 \\
28.1 \%\end{array}$ & $\begin{array}{c}26 \\
17.8 \%\end{array}$ & $\begin{array}{c}13 \\
8.9 \%\end{array}$ & 16.621 & .002 \\
\hline $\begin{array}{l}3 . \quad \text { Organization } \\
\text { atmosphere is full of } \\
\text { spirit and cooperation }\end{array}$ & $\begin{array}{c}25 \\
17.2 \%\end{array}$ & $\begin{array}{c}35 \\
24.0 \%\end{array}$ & $\begin{array}{c}37 \\
25.3 \%\end{array}$ & $\begin{array}{c}30 \\
20.5 \%\end{array}$ & $\begin{array}{c}19 \\
13.0 \%\end{array}$ & 8.207 & .084 \\
\hline $\begin{array}{l}\text { 4. Your work is always } \\
\text { meaningful }\end{array}$ & $\begin{array}{c}25 \\
17.1 \%\end{array}$ & $\begin{array}{c}44 \\
30.1 \%\end{array}$ & $\begin{array}{c}29 \\
19.9 \%\end{array}$ & $\begin{array}{c}34 \\
23.3 \%\end{array}$ & $\begin{array}{c}14 \\
9.6 \%\end{array}$ & 18.000 & .001 \\
\hline $\begin{array}{l}\text { 5. Your organization } \\
\text { encourages creativity }\end{array}$ & $\begin{array}{c}28 \\
19.2 \%\end{array}$ & $\begin{array}{c}37 \\
25.3 \%\end{array}$ & $\begin{array}{c}32 \\
21.9 \%\end{array}$ & $\begin{array}{c}33 \\
22.6 \%\end{array}$ & $\begin{array}{c}16 \\
11.0 \%\end{array}$ & 9.862 & .043 \\
\hline
\end{tabular}

The p-value of all the statements in Table 6 was less than .05 except statement NO.3 whose value (.084) was more than .05. So, eliminating that statement, the analysis was carried out again and the result was as follows: 
Table 7 Employee Engagement

\begin{tabular}{|c|c|c|c|c|c|c|c|c|}
\hline Statements & SD & D & $\mathbf{N}$ & $\mathbf{A}$ & SA & $\begin{array}{c}\text { KS } \\
\text { value }\end{array}$ & $\chi^{2}$ & $\begin{array}{c}\text { p- } \\
\text { value }\end{array}$ \\
\hline $\begin{array}{l}\text { 1. The top management } \\
\text { supports employees to } \\
\text { achieve continuous } \\
\text { improvement }\end{array}$ & $\begin{array}{c}29 \\
19.9 \%\end{array}$ & $\begin{array}{c}29 \\
19.9 \%\end{array}$ & $\begin{array}{c}47 \\
32.1 \%\end{array}$ & $\begin{array}{c}23 \\
15.8 \%\end{array}$ & $\begin{array}{c}18 \\
12.3 \%\end{array}$ & .166 & \multirow{4}{*}{74.034} & \multirow{4}{*}{.000} \\
\hline $\begin{array}{ll}2 . \quad \text { The } & \text { organization } \\
\text { provides } & \text { professional } \\
\text { training } & \\
\end{array}$ & $\begin{array}{c}31 \\
21.2 \%\end{array}$ & $\begin{array}{c}35 \\
24.0 \%\end{array}$ & $\begin{array}{c}41 \\
28.1 \%\end{array}$ & $\begin{array}{c}26 \\
17.8 \%\end{array}$ & $\begin{array}{c}13 \\
8.9 \%\end{array}$ & .176 & & \\
\hline $\begin{array}{l}\text { 4. Your work is always } \\
\text { meaningful }\end{array}$ & $\begin{array}{c}25 \\
17.1 \%\end{array}$ & $\begin{array}{c}44 \\
30.1 \% \\
\end{array}$ & $\begin{array}{c}29 \\
19.9 \%\end{array}$ & $\begin{array}{c}34 \\
23.3 \%\end{array}$ & $\begin{array}{c}14 \\
9.6 \%\end{array}$ & .176 & & \\
\hline $\begin{array}{l}\text { 5. Your organization } \\
\text { encourages creativity }\end{array}$ & $\begin{array}{c}28 \\
19.2 \% \\
\end{array}$ & $\begin{array}{c}37 \\
25.3 \% \\
\end{array}$ & $\begin{array}{c}32 \\
21.9 \%\end{array}$ & $\begin{array}{c}33 \\
22.6 \% \\
\end{array}$ & $\begin{array}{c}16 \\
11.0 \%\end{array}$ & .193 & & \\
\hline
\end{tabular}

Null Hypothesis 2: No relationship exists between the VM tools and the choices of the respondents.

Table 7 shows p-value $<0.05$. So the null hypothesis 2 got rejected. i.e., it is understood that there exists a significant relationship between employee engagement and the choices of the respondents. From K-S values of Kolmogorov-Smirnov test, 'Your organization encourages creativity' ranked first (0.193); both 'The organization provides professional training' and 'Your work is always meaningful' ranked second $(0.176)$ and 'The top management supports employees to achieve continuous improvement' ranked third $(0.166)$.

Table 8 Leadership Style adopted by the companies

\begin{tabular}{|l|c|c|}
\hline \multicolumn{1}{|c|}{ Leadership Style } & Frequency & \% \\
\hline Top Down & 37 & 25.3 \\
\hline Bottom Up & 60 & 41.1 \\
\hline Participative & 4 & 33.6 \\
\hline
\end{tabular}

Table 8 shows the various Leadership style adopted to ensure the successful implementation of CI in their companies.

Table 9 Leadership Practices

\begin{tabular}{|c|c|c|c|c|c|c|c|c|}
\hline Statements & SD & D & $\mathbf{N}$ & $\mathbf{A}$ & SA & $\begin{array}{c}\text { KS } \\
\text { value }\end{array}$ & $\chi^{2}$ & $\begin{array}{c}\text { p- } \\
\text { value }\end{array}$ \\
\hline $\begin{array}{l}\text { 1. The organization has } \\
\text { a kaizen team leader }\end{array}$ & $\begin{array}{c}31 \\
21.2 \%\end{array}$ & $\begin{array}{c}27 \\
18.5 \%\end{array}$ & $\begin{array}{c}47 \\
32.2 \%\end{array}$ & $\begin{array}{c}25 \\
17.1 \%\end{array}$ & $\begin{array}{c}16 \\
11.0 \%\end{array}$ & .174 & \multirow{5}{*}{42.667} & \multirow{5}{*}{.001} \\
\hline $\begin{array}{l}\text { 2. The top management } \\
\text { supports the change } \\
\text { journey }\end{array}$ & $\begin{array}{c}33 \\
22.6 \%\end{array}$ & $\begin{array}{c}36 \\
24.7 \%\end{array}$ & $\begin{array}{c}33 \\
22.6 \%\end{array}$ & $\begin{array}{c}34 \\
23.3 \%\end{array}$ & $\begin{array}{c}10 \\
6.8 \%\end{array}$ & .178 & & \\
\hline $\begin{array}{l}\text { 3. The leaders ensure } \\
\text { that every staff job can } \\
\text { add value to the } \\
\text { customers }\end{array}$ & $\begin{array}{c}27 \\
18.5 \%\end{array}$ & $\begin{array}{c}32 \\
21.8 \%\end{array}$ & $\begin{array}{c}45 \\
30.8 \%\end{array}$ & $\begin{array}{c}29 \\
19.8 \%\end{array}$ & $\begin{array}{c}13 \\
8.1 \%\end{array}$ & .168 & & \\
\hline $\begin{array}{l}\text { 4. The team leaders } \\
\text { provide effective } \\
\text { communication between } \\
\text { the staff }\end{array}$ & $\begin{array}{c}23 \\
15.7 \%\end{array}$ & $\begin{array}{c}41 \\
28.1 \%\end{array}$ & $\begin{array}{c}36 \\
24.7 \%\end{array}$ & $\begin{array}{c}27 \\
18.5 \%\end{array}$ & $\begin{array}{c}18 \\
13.0 \%\end{array}$ & .188 & & \\
\hline $\begin{array}{l}\text { 5. The leaders support } \\
\text { Gemba commitment for } \\
\text { continuous } \\
\text { improvement } \\
\text { achievement }\end{array}$ & $\begin{array}{c}30 \\
20.5 \%\end{array}$ & $\begin{array}{c}33 \\
22.6 \%\end{array}$ & $\begin{array}{c}46 \\
31.5 \%\end{array}$ & $\begin{array}{c}22 \\
15.1 \%\end{array}$ & $\begin{array}{c}15 \\
10.3 \%\end{array}$ & .164 & & \\
\hline
\end{tabular}


Null Hypothesis.3: No relationship exists between the Leadership Practices and the choices of the respondents.

Table 9 shows p-value $<0.05$. So the null hypothesis 3 got rejected. i.e., it is understood that there exists a significant relationship between the Leadership Practices and the choices of the respondents. From K-S values of Kolmogorov-Smirnov test, 'The team leaders provide effective communication between the staff' ranked first (0.188); 'The top management supports the change journey' ranked second (0.178) and 'The organization has a kaizen team leader' (0.174).

Table 10 Risk Management Tools adopted by the companies

\begin{tabular}{|l|c|c|}
\hline \multicolumn{1}{|c|}{ Risk Management Tools } & Frequency & \% \\
\hline HAZOP & 41 & 28.1 \\
\hline Process FMEA & 28 & 19.2 \\
\hline Ishikawa Diagrams & 50 & 34.2 \\
\hline Fault Tree Analysis & 27 & 18.5 \\
\hline
\end{tabular}

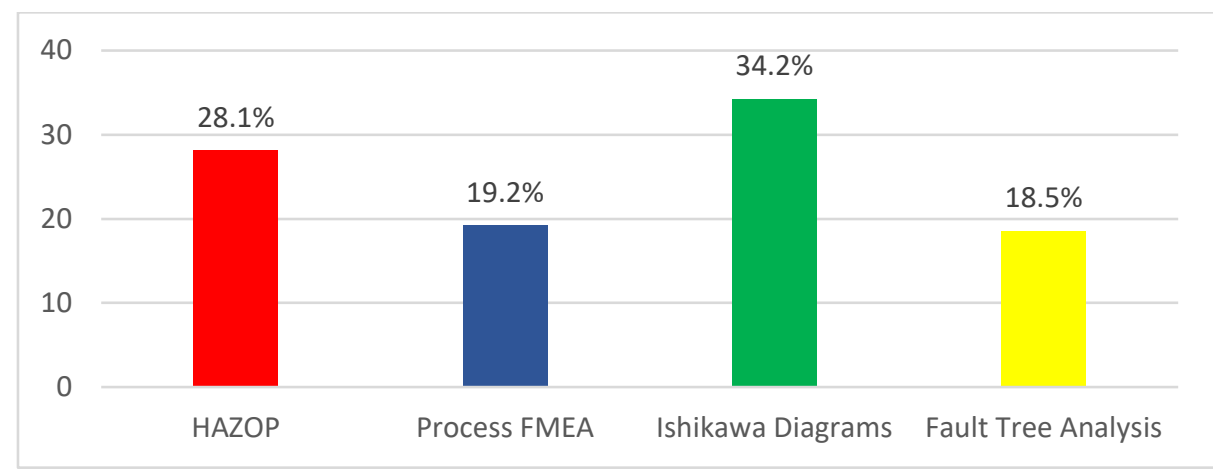

Figure 4 Risk Management Tools adopted by the companies

Figure 4 shows the various Risk Management tools used to ensure successful implementation of CI in their companies and the popular tool among the respondents i.e. Ishikawa Diagrams.

Table 11 Risk Management (RM) Tools

\begin{tabular}{|c|c|c|c|c|c|c|c|c|}
\hline Statements & SD & D & $\mathbf{N}$ & $\mathbf{A}$ & $\mathbf{S A}$ & $\begin{array}{c}\text { KS } \\
\text { value }\end{array}$ & $\chi^{2}$ & $\begin{array}{c}\mathbf{p} \\
\text { value }\end{array}$ \\
\hline $\begin{array}{l}\text { 1. The organization has } \\
\text { an effective RM team }\end{array}$ & $\begin{array}{c}25 \\
17.0 \%\end{array}$ & $\begin{array}{c}43 \\
29.5 \%\end{array}$ & $\begin{array}{c}47 \\
32.2 \%\end{array}$ & $\begin{array}{c}20 \\
13.7 \%\end{array}$ & $\begin{array}{c}11 \\
7.6 \%\end{array}$ & .182 & \multirow{5}{*}{55.444} & \multirow{5}{*}{+.000} \\
\hline $\begin{array}{l}\text { 2. The RM is applied to } \\
\text { achieve a high level of } \\
\text { reliability and } \\
\text { profitability }\end{array}$ & $\begin{array}{c}26 \\
17.8 \%\end{array}$ & $\begin{array}{c}40 \\
27.4 \%\end{array}$ & $\begin{array}{c}33 \\
22.6 \%\end{array}$ & $\begin{array}{c}31 \\
21.2 \%\end{array}$ & $\begin{array}{c}16 \\
11.0 \%\end{array}$ & .190 & & \\
\hline $\begin{array}{l}\text { 3. The risk management } \\
\text { plan allows you to } \\
\text { identify and treat the } \\
\text { risk efficiently }\end{array}$ & $\begin{array}{c}35 \\
24.0 \%\end{array}$ & $\begin{array}{c}42 \\
28.7 \%\end{array}$ & $\begin{array}{c}37 \\
25.3 \%\end{array}$ & $\begin{array}{c}23 \\
15.8 \%\end{array}$ & $\begin{array}{c}9 \\
6.2 \%\end{array}$ & .194 & & \\
\hline $\begin{array}{l}\text { 4. The risk management } \\
\text { process eliminates non } \\
\text { value added activities }\end{array}$ & $\begin{array}{c}21 \\
14.3 \%\end{array}$ & $\begin{array}{c}44 \\
30.1 \%\end{array}$ & $\begin{array}{c}49 \\
33.6 \%\end{array}$ & $\begin{array}{c}21 \\
14.4 \%\end{array}$ & $\begin{array}{c}11 \\
7.6 \%\end{array}$ & .183 & & \\
\hline $\begin{array}{l}\text { 5. The RM plan } \\
\text { identifies roles and } \\
\text { responsibilities towards } \\
\text { risk }\end{array}$ & $\begin{array}{c}23 \\
15.8 \%\end{array}$ & $\begin{array}{c}47 \\
32.2 \%\end{array}$ & $\begin{array}{c}43 \\
29.5 \%\end{array}$ & $\begin{array}{c}19 \\
13.0 \%\end{array}$ & $\begin{array}{c}14 \\
9.5 \%\end{array}$ & .201 & & \\
\hline
\end{tabular}


Null Hypothesis.4: No relationship exists between the Leadership Practices and the choices of the respondents.

Table 11 shows p-value $<0.05$. So the null hypothesis 4 got rejected. i.e., it is understood that there exists a significant relationship between the Risk Management Tools and the choices of the respondents. From the K-S values of Kolmogorov-Smirnov test, 'The RM plan identifies roles and responsibilities towards risk' ranked first (0.201); 'The risk management plan allows you to identify and treat the risk efficiently' ranked second (0.194) and 'The risk management process eliminates non-value-added activities ranked third $(0.183)$.

\section{Discussions}

\section{Average Sales}

About the company average sales, the majority of the respondents $(67.1 \%)$ declared that the average sales had increased over the past three financial years (2017-2020) whereas $25.3 \%$ of the respondents reported that the average sales were constant over the past three financial years. $7.5 \%$ of the respondents reported that their company's average sales had decreased over the past three financial years.

\section{Decrease in Average Cycle Time}

The majority of the respondents $(58.2 \%)$ reported that the average cycle time decreased at their site, whereas $23.3 \%$ of the respondents were unsure of the same. $18.5 \%$ of the respondents disagreed that average cycle time decreased at their site.

\section{Recalling of Product in Site (Including Voluntarily)}

A majority of $45.9 \%$ respondents agreed that their site recalled the product in the last financial year (2018 2019) whereas $32.9 \%$ of the respondents were against the fact that their site recalled the product and $21.2 \%$ were unsure of the fact that their site recalled the product.

\section{Preferred Critical Success Factors of the Companies}

Among the VM tools, the respondents preferred 'VM strategy enables the flow of information' followed by 'VM leads to providing the best solution', and 'VM tools allow you to detect the deviations from the standards' and 'VM facilitates the distribution of responsibilities among employees' ranked third.

Among the employee engagement factors, the respondents chose 'Organization encourages creativity' followed by both 'The organization provides professional training', 'Your work is always meaningful', 'VM tools allow you to detect the deviations from the standards', and 'The top management supports employees to achieve continuous improvement'.

Among the leadership practices, the respondents selected 'The team leaders provide effective communication between the staff' followed by 'The top management supports the change journey'. 'VM tools allow you to detect the deviations from the standards', and 'The organization has a kaizen team leader'.

Among the risk management tools, the respondents selected 'The RM plan identifies roles and responsibilities towards risk' followed by 'The risk management plan allows you to identify and treat the risk efficiently', and 'The risk management process eliminates non-value-added activities'.

\section{Savings of the companies due to continuous improvement projects}

$35.6 \%$ of respondents have recorded that their company has received more than OMR 1,00,000 annual savings from continuous improvement projects, $32.4 \%$ have recorded that their company has received less than OMR 10,000 annual savings from continuous improvement projects, 21.9 percent respondents have recorded that their company has received savings between OMR 10,001 - OMR 25,000 and 11percent respondents have recorded that their company has received savings between OMR 50,001 - OMR 1,00,000 in the financial year 2018-2019.

\section{Conclusion}

The overall sales have risen, while the average processing time has declined and also the product recalls have risen. The majority of the manufacturing firms were using only the CI program for the past five years. However, since these projects took an average of 7 years to execute, there were not substantial cost savings for these businesses. The overall sales increased, while the average processing time had declined and the product recalls also had increased. 
It was observed that VM strategy enables the flow of information and provides the best solutions in detecting deviations from the standards. It was also observed that the top management supports employees to achieve continuous improvement through effective communication. The important risk management tools were risk management plans to identify and treat the risk efficiently. Therefore, the most important factors for the effective implementation of $\mathrm{CI}$ in industrial firms were effective communication, top management encouragement, and employee involvement, and a conducive organizational atmosphere.

This study identified the use of CI tools through the survey from the managers of the production units in the manufacturing industry. All critical success factors assessed in this study influenced the company's decision to implement CI. These critical success factors, combined with the continuous improvement realized by manufacturing companies, strongly suggested that the continuous improvement tools are an integral component of business strategy. Organization atmosphere, Top management, Effective communication, and Organization creativity are the important critical success factor in CI implementation. The results of this study were similar to the results of the study by Antony et al. (2015) in which similar two factors were found to be influential in small and medium-sized manufacturing enterprises in the UK. Verification of these results with a larger, more representative sample of the Omani manufacturing industry is recommended.

\section{Acknowledgment}

The research was carried out with funding from The Research Council, Oman (TRC) - Ministry of Higher Education Research \& Innovation of the Sultanate of Oman under the Block Funding Program vide No. TRC/BFP/SHCT/01/2019.

\section{References}

1. Abrahamsson, G. \& Gerdin, J. (2006). Exploiting institutional contradictions: the role of management accounting in continuous improvement implementation. Qualitative Research in Accounting \& Management, 3(2), 126-144. https://doi.org/10.1108/11766090610670668

2. Al-Khawaldeh, K. \& Sloan, T. (2007). Continuous Improvement in Manufacturing Companies. Jordan. International Journal of Technology Management, 37(3-4), 323-331.

3. Anand, G., Ward, P. T., Tatikonda, M. V. \& Schilling, D. A. (2009). Dynamic Capabilities through Continuous Improvement Infrastructure. Journal of operations management, 27(6), 444-461. https://doi.org/10.1016/j.jom.2009.02.002

4. Antony, J., Kumar, M. \& Tiwari, M. K. (2005). An Application of Six Sigma Methodology to Reduce the Engine-Overheating Problem in an Automotive Company. Proceedings of the Institution of Mechanical Engineers, Part B: Journal of Engineering Manufacture, 219(8), 633-646.

5. Atkinson, C. (1994). Continuous improvement: The ingredients of change. International Journal of Contemporary Hospitality Management, 6(1/2), 06-08. https://doi.org/10.1108/09596119410052017

6. Augsdorfer, P. \& Harding, R. (1995). Changing competitive forces in Europe continuous improvement in a sample of French, German and British companies. European Business Review, 95(4), 3-9. https://doi.org/10.1108/09555349510085209

7. Avanesov, E. (2009, November). Risk Management in ISO 9000 Series Standards. International conference on risk assessment and Management, 24-25 ${ }^{\text {th }}$ Nov.2009, Geneva.

8. Bechet, T. P. (2000). Developing Staffing Strategies That Work: Implementing Pragmatic, Nontraditional approaches. Public Personnel Management, 29(4), 465-477. https://doi.org/10.1177/009102600002900405

9. Bessant, J. \& Francis, D. (1999). Developing Strategic Continuous Improvement Capability. International Journal of Operations \& Production Management. 19(11), 1106-1119. https://doi.org/10.1108/01443579910291032

10. Caffyn, S. (1999). Development of a continuous improvement self-assessment tool. International Journal of Operations \& Production Management, $19 \quad$ (11), 1138-1153. https://doi.org/10.1108/01443579910291050

11. Chapman, R. L. \& Hyland, P. W. (1997). Continuous Improvement Strategies Across Selected Australian Manufacturing Sectors. Benchmarking for Quality Management \& Technology, 4(3), 175188. https://doi.org/10.1108/14635779710181415

12. Chen, J. C., Dugger, J. \& Hammer, B. (2001). A Kaizen Based Approach for Cellular Manufacturing System Design: A Case Study. 27(2). https://doi.org/10.21061/jots.v27i2.a.3 
13. Choi, T. M., Chiu, C. H., \& Chan, H. K. (2016). Risk Management of Logistics Systems, 90,1-6. https://doi.org/10.1016/j.tre.2016.03.007

14. Corso, M., Giacobbe, A., Martini, A. \& Pellegrini, L. (2007). Tools and abilities for continuous improvement: what are the drivers of performance? International Journal of Technology Management, 37(3-4), 348-365. https://doi.org/10.1504/IJTM.2007.012268

15. Dabhilkar, M. \& Bengtsson, L. (2004). Balanced Scorecards for Strategic and Sustainable Continuous Improvement Capability. Journal of Manufacturing Technology Management, 15(4), 350-359. https://doi.org/10.1108/17410380410535053

16. Danciulescu, A. (2013). The Role of Risk Management and Treatment Methods Applied in Today's Economy. Romanian Economic and Business Review-Special Issue, 18-25.

17. Das, A., Kumar, V., \& Kumar, U. (2011). The role of leadership competencies for implementing TQM. International Journal of Quality \& Reliability Management, 28(2), 195-219. https://doi.org/10.1108/02656711111101755

18. Delbridge, R. \& Barton, H. (2002). Organizing for Continuous Improvement. International Journal of Operations \& Production Management, 22(6), 680-692. https://doi.org/10.1108/01443570210427686

19. De Morais, T. C. M., Gomes, M. D. L. B. \& Da Silva, L. B. (2012). Skills for Driving Continuous Improvement in an Electricity Distributor. International Journal of Basic \& Applied Sciences, 12(03), $17-24$.

20. Dossenbach, T. (2006). Implementing Total Productive Maintenance. Wood and Wood Products, 111(2), 29-32.

21. Escrig-Tena, A. B. (2004). TQM as a competitive factor. International Journal of Quality \& Reliability Management, 21(6), 612-637. https://doi.org/10.1108/02656710410542034

22. Galli, B. J. (2018). Continuous Improvement Relationship to Risk Management: The Relationship Between them. International Journal of Applied Management Sciences and Engineering, 5(2), 1-14. http://doi.org/10.4018/IJAMSE.2018070101

23. Galli, B. J. \& Kaviani, M. A. (2017). Are Project Management and Project Life Cycles Affected by Marketing and New Product Development? The Journal of Modern Project Management, 5(1). http://doi.org/10.19255/JMPM01303

24. Harris, C. \& Harris, R. (2008). Lean Connections: Making Information Flow Efficiently and Effectively. CRC Press.

25. Hyland, P., Mellor, R., O’Mara, E. \& Kondepudi, R. (2000). A Comparison of Australian Firms and their Use of Continuous Improvement Tools. The TQM Magazine. https://doi.org/10.1108/09544780010318370

26. Jabnoun, N. (2001). Values underlying continuous improvement. The TQM Magazine. https://doi.org/10.1108/09544780110406137

27. Lee, H. L. (2004). The triple-A supply chain. Harvard Business Review, 82(10), 102-113.

28. Liker, J. K. \& Hoseus, M. (2008). Culture - The Heart and Soul of the Toyota Way. Tata McGraw-Hill, New Delhi

29. Maskell, B. H., Baggaley, B. \& Grasso, L. (2011). Practical Lean Accounting: A Proven System for Measuring and Managing the Lean Enterprise. CRC Press.

30. Marin-Garcia, J. A., del Val, M. P. \& Martín, T. B. (2008). Longitudinal Study of the Results of Continuous Improvement in an Industrial Company. Team Performance Management: An International Journal, 14(1/2), 56-69. https://doi.org/10.1108/13527590810860203

31. Nunnally, J. (1960). The Place of Statistics in Psychology. Educational and Psychological Measurement, 20(4), 641-650. https://doi.org/10.1177/001316446002000401

32. Oman Observer (2020, 16 ${ }^{\text {th }}$ Dec.2020). Vision 2040 to Improve Oman's Global Standings. https://www.omanobserver.om/vision-2040-to-improve-omans-global-standings/

33. Rad, A. M. M. (2006). The Impact of Organizational Culture on the Successful Implementation of Total Quality Management. The TQM Magazine. https://doi.org/10.1108/09544780610707101

34. Singh, J. \& Singh, H. (2018). Enigma of KAIZEN approach in manufacturing industry of Northern India-a case study. International Journal of Quality \& Reliability Management, 35(1), 187-207. https://doi.org/10.1108/IJQRM-12-2016-0220

35. Soltani, E. (2005). Top Management: A Threat or an Opportunity to TQM? Total Quality Management \& Business Excellence, 16(4), 463-476. https://doi.org/10.1080/14783360500078441

36. Talib, F., Rahman, Z. \& Qureshi, M. N. (2011). A Study of Total Quality Management and Supply Chain Management Practices. International Journal of Productivity and Performance Management, 60(3), 268-288. https://doi.org/10.1108/17410401111111998 
37. Taneja, S., Sewell, S. S. \& Odom, R. Y. (2015). A Culture of Employee Engagement: A Strategic Perspective for Global Managers. Journal of Business Strategy. 36(3), 46-56. https://doi.org/10.1108/JBS-06-2014-0062

38. Tezel, A., Koskela, L. J., Tzortzopoulos-Fazenda, P., Formoso, C. T., Alves, T. D. C. L., Barros Neto, J. D. P., Viana, D. \& Mota, B. (2010). Process Transparency On Construction Sites: Examples from Construction Companies in Brazil. Annual Conference of the International Group for Lean Construction, 296-305. http://www.repositorio.ufc.br/handle/riufc/6070

39. Tezel, A., Koskela, L., \& Tzortzopoulos, P. (2016). Visual Management in Production Management: A Literature Synthesis. Journal of Manufacturing Technology Management, 27(6), 766-799. https://doi.org/10.1108/JMTM-08-2015-0071

40. Venkateswarlu, P. \& Nilakant, V. (2005). Adoption and Persistence of TQM Programmes-Case Studies of Five New Zealand Organizations. Total Quality Management \& Business Excellence, 16(7), 807-825. https://doi.org/10.1080/14783360500077310

41. Welo, T. \& Ringen, G. (2015). Investigating Lean development practices in SE companies: A comparative study between sectors. Procedia Computer Science, 44, 234-243. https://doi.org/10.1016/j.procs.2015.03.056

42. World Economic Forum (2021). Readiness for the Future of Production. World Economic Forum. https://www.weforum.org/projects/readiness-for-the-future-of-production-country-profiles

43. Yanjiang, C., Dan, W. \& Lang, X. (2006, June). Influencing factors of continuous improvements and tendency of change. In 2006 IEEE International Conference on Management of Innovation and Technology, 1, 181-185. https://doi.org/10.1109/ICMIT.2006.262259

Copyright: () 2021 by the authors. Licensee Global Scientific Publications, Oman.

This work is licensed under a Creative Commons Attribution-ShareAlike 4.0 International License.

This is an open-access journal and the articles published in this journal are distributed under the terms of CC-BY-SA. 